\title{
Niederschlagsbilder von Gasströmungen durch Thermodiffusion
}

\author{
Von Klaus Clusius und Horst Meyer \\ Aus dem Physikalisch-Chemischen Institut der Universität Zürich \\ (Z. Naturforschg. 6 a, 401-402 [1951]; eingegangen am 1. Mai 1951) \\ John Eggert zum 60. Geburtstag gewidmet
}

\begin{abstract}
Es werden Versuche mitgeteilt, um die durch eine Wasserstoff-Flamme hervorgerufenen Konvektionsströmungen bei Drucken von 30 bis $200 \mathrm{~mm} \mathrm{Hg}$ durch Niederschläge von verdampftem Salz scharf sichtbar zu machen und so zu fixieren.
\end{abstract}

1. Die Staubabscheidung durch Thermodiffusion ist eine im täglichen Leben weit verbreitete Erscheinung ${ }^{1}$. Staub, Rauch und andere in der Luft befindliche Schwebeteilchen, die einem Temperaturgradienten ausgesetzt sind, wandern in der Richtung der fallenden Temperatur. Deshalb schwärzen sich Decken und Wände über einer Heizung oder einem Dampfrohr dort, wo sie vom aufsteigenden, warmen Luftstrom getroffen werden. Die suspendierten Teilchen streben nach der kalten Wand, an der sie sich niederschlagen. In Fabriken und Laboratorien zeichnet sich im Laufe der Jahre auf der Innenseite der Wände die eiserne Gebäudekonstruktion dunkel ab, da infolge der guten Wärmeleitfähigkeit des Metalls die Wand im Winter dort kälter ist als die Umgebung, so daß an diesen Stellen die Luft einem größeren Temperaturgradienten ausgesetzt ist. Die erzeugten Niederschläge sind gewöhnlich diffus und an den Orten höherer Temperaturgradienten besonders ausgeprägt (Abb. $\left.1 \mathrm{u} .2^{*}\right)$.

2. Wir haben neuerdings mit einer Anordnung, in der die Verbrennung von Wasserstoff bei Unterdruck untersucht wurde, recht scharfe Niederschlagsbilder von Salznebeln durch Thermodiffusion erhalten, die eine deutliche Vorstellung von den im Gasraum herrschenden Strömungsverhältnissen geben.

Die Verbrennungskammer besteht aus einer Glasglocke von $\sim 1,5 l$ Inhalt, die auf einen kräftigen, eisernen Teller aufgeschliffen ist $\left(\mathrm{Abb} .3^{*}\right)$. Die Verbrennungswärme wird durch eine kupferne, von $12^{\circ}$ kaltem Wasser durchströmte Kühlschlange abgeführt. An ihr schlägt sich zugleich der gebildete Wasserdampf nieder, so daß die Glocke während der Versuche klar von Feuchtigkeit bleibt. Die Gase strömen im stöchiometrischen Verhältnis durch Strömungsmesser und Hahn- und Kapillarsysteme zur passenden Druckredu-

* Abb. 1 u. 2, s. Tafel S. 400 a; Abb. 4-6, s. Tafel S. 400 b.

$1 \mathrm{~K}$. Clu s i us, Z. Ver. dtsch. Ing., Beih. Verfahrenstechnik 1941, Nr.2, S. 23; siehe auch die Bemerkung von T. L. I b b s, Trans. Faraday Soc. 32, 1082 [1936]. zierung. Während der Sauerstoff unmittelbar in die Glocke tritt, verbrennt der Wasserstoff an einer Pyrex-Düse, deren Außenseite und Rand vorher im Gebläse mit geschmolzenem Kochsalz überkrustet worden sind.

Die Glocke wird bis zu einem Druck von etwa $10 \mathrm{~mm} \mathrm{Hg}$ mit Helium und Sauerstoff gefüllt, der Wasserstoffstrom angestellt und durch einen Funkenstrom bis zum Bestehen einer stationären F'lamme gezündet. Bei größerem Unterdruck sind die anfänglich auftretenden Explosionen harmlos. Erhöht man die Sauerstoffkonzentration, so zieht sich die F'lamme stark zusammen, weil die Verbrennungsgeschwindigkeit zunimmt. Die Reaktionswärme verteilt sich dann auf eine kleinere Fläche und reicht aus, um den Salzbelag am Düsenrand zu schmelzen und zu verdampfen. Der Salzdampf wird von der entstehenden Konvektionsströmung mitgenommen und kondensiert sich erst wieder in einiger Entfernung von der Flamme zu einem Aerosol. Dieses schlägt sich größtenteils an der Glocke auf der dem Kühler abgewandten Seite nieder. Manchmal dient auch eine vertikale Glasplatte zwischen Flamme und Kühler als Auffänger. Bei den niedrigsten Drucken von 30 bis $40 \mathrm{~mm} \mathrm{Hg}$ kann man auf den Heliumzusatz, der die Diffusion verbessern soll, verzichten. Hier verhalten sich nämlich alle Gase praktisch gleich, da der Sauerstoffpartialdruck ohnehin nicht unter einen bestimmten Grenzwert sinken darf und das Restgas überwiegend aus Wasserdampf besteht. Brauchbare „Bilder" zeigen sich nach $1 / 2$ bis 2 Minuten, worauf die Gasströme abgestellt und die Niederschläge photographiert werden.

3. Bei hohen, in der Nähe von Atmosphärendruck liegenden Drucken zeigen die Abscheidungen das übliche Aussehen, indem sie im Oberteil der Glocke einen gleichmäßigen, diffusen Beschlag bilden. Geht man aber zu Drucken von $100 \mathrm{~mm} \mathrm{Hg}$ und tiefer über, 
so ändert sich die Struktur des Belags völlig (Abb. 4 bis $\left.7^{*}\right)$. Man erkennt dann deutlich das Vorliegen der aufsteigenden Ströme über der Düse, die durch die obere Begrenzung des Gefäßes in zwei sich einrol-

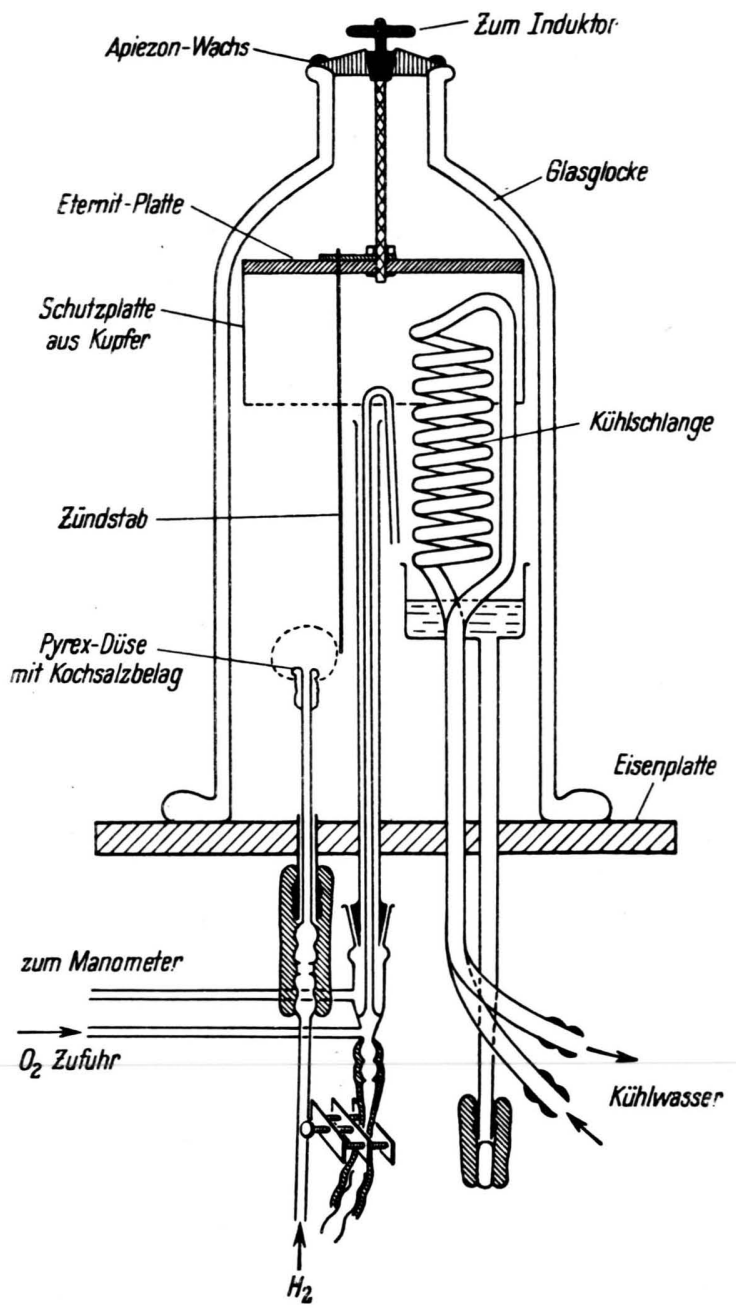

Abb. 3. Anordnung zur Untersuchung von $\mathrm{H}_{9}$-Flammen und Salzniederschlägen bei Unterdruck; Maßstab 1:3.

lende Wirbel zerlegt wird. Diese Struktur wird durch die Form des Versuchsgefäßes mit dem seitlich angeordneten Kühler hervorgerufen.

$\mathrm{Zu}$ einem qualitativen Verständnis der notwendigen Bedingungen, die zu scharf begrenzten, zart strukturierten Niederschlagsbildern führen, gelangt man auf folgendem Wege: Die innere Reibung der Gase ist in dem hier in Betracht kommenden Bereich kaum vom Druck abhängig, so daß die Konvektionsgeschwindigkeit der heißen Flammengase in erster Näherung dem Druck proportional ist. Tatsächlich

* Abb. 4-6, s. Tafel S. 400 b; Abb. 7, s. Tafel S. 400 a. kann man bei Drucken von 30 bis $40 \mathrm{~mm} \mathrm{Hg}$ in geeigneter Beleuchtung die Bildung der Nebelfäden in einiger Entfernung über der Flamme und ihre Zirkulation im Gasraum bequem mit dem Auge verfolgen. Bei hohen Drucken ist dies dagegen unmöglich, da eine viel zu schnelle Konvektion verbunden mit einer energischen Durchwirbelung statthat. Ferner ist mit sinkendem Druck die Diffusion, die dem Druck umgekehrt proportional ist, stark heraufgesetzt, bei 30 bis $50 \mathrm{~mm} \mathrm{Hg}$ etwa auf das 20 -fache gegenüber den Verhältnissen bei Atmosphärendruck. Beide Effekte, die verminderte Konvektion und die gesteigerte Diffusion, wirken also in der gleichen Richtung so, daß sich bei kleinen Drucken der Einfluß der Diffusion gegenüber dem der Konvektion überwiegend geltend macht. Die Salzteilchen setzen sich dann aus den langsam zirkulierenden heißen Verbrennungsgasen auf der kalten Wand durch Thermodiffusion so rasch ab, daß die Struktur der Strömung noch im Niederschlag in Einzelheiten sichtbar und somit fixiert wird. Unsymmetrien in der Dichte des Belages sind vorwiegend durch ungleichmäßige Verdampfung des Salzes bedingt.

Mit der voranstehenden Erklärung kann man den Einfluß des Druckes auf das Aussehen des Niederschlages qualitativ gut verstehen. Beim kleinsten Druck (30 bis $40 \mathrm{~mm} \mathrm{Hg}$ ) ist der mittlere Diffusionsweg am größsten; entsprechend beginnt auf Abb. 4 der Niederschlag schon unterhalb des Düsenrandes, er ist im aufsteigenden Teil breit angelegt, und die Konvektion setzt sich nicht bis in den Oberteil der Glocke durch. Bei Abb. 5 mit $90 \mathrm{~mm} \mathrm{Hg}$ erfolgt die Abscheidung erst oberhalb der Düse, der aufsteigende Gasstrom erscheint schmaler und gelangt bis in den Oberteil der Glocke. Bei 200 mm Druck auf Abb. 6 ist der mittlere Diffusionsweg bereits kleiner als dem $\mathrm{Ab}$ stand Düse-Glaswand $(3 \mathrm{~cm})$ entspricht; die rasche Konvektion trägt den Salznebel bis hoch in die Glocke hinauf, die unten beschlagfrei bleibt, und es sind nur noch schwache Reste der Strömungsstruktur zu erkennen. Bei weiterer Drucksteigerung ändert sich das Bild nur noch dahin, daß der Belag ganz diffus wird.

4. Der vorliegenden Darstellung entspricht auch die Form der Flamme, worauf wir an anderer Stelle zurückkommen werden ${ }^{2}$. Die in ästhetischer Hinsicht recht befriedigend anmutenden "Salzbilder" regen dazu an, die Strömungsverhältnisse im Bereich kleiner Drucke noch bei anderen Vorgängen, z. B. in Injektordüsen von Dampfstrahlpumpen, bequem sichtbar zu machen.

2 Erscheint in Helv. chim. Acta. 